\title{
L'échec de Caliban : rationalités et altérités dans le monde ibérique et latino-américain
}

\section{Enrique Sanchez Albarracin}

\section{(2) OpenEdition}

1 Journals

\section{Édition électronique}

URL : https://journals.openedition.org/cher/3470

DOI : $10.4000 /$ cher.3470

ISSN : 2803-5992

\section{Éditeur}

Presses universitaires de Strasbourg

\section{Édition imprimée}

Date de publication : 1 décembre 2015

Pagination : 39-52

ISBN : 978-2-86820-913-9

ISSN : 1968-035X

\section{Référence électronique}

Enrique Sanchez Albarracin, «L'échec de Caliban : rationalités et altérités dans le monde ibérique et latino-américain », reCHERches [En ligne], 15 | 2015, mis en ligne le 01 décembre 2021, consulté le 19 décembre 2021. URL : http://journals.openedition.org/cher/3470; DOI : https://doi.org/10.4000/cher. 3470

\section{(c) (i) (3) (-)}

Ce(tte) œuvre est mise à disposition selon les termes de la Licence Creative Commons Attribution -

Pas d'Utilisation Commerciale - Partage dans les Mêmes Conditions 4.0 International. 


\title{
L'échec de Caliban: rationalités et altérités dans le monde ibérique et latino-américain
}

\author{
Enrioue Sanchez Albarracin \\ Université Lumière Lyon 2
}

\begin{abstract}
El pensamiento es un fluido de ideas que viaja en la historia a través de crisis internas, de obstáculos epistemológicos, de cambios paradigmáticos, de resignificaciones teóricas; pero que no permite hacer cortes temporales absolutos; donde el conocimiento que busca la unidad, la generalidad y la transdisciplinariedad, tampoco puede escapar a la identidad propia de cada paradigma científico y al campo específico en el que una teoría produce conocimientos, verdades y sentidos. Donde la verdad nunca es absoluta, ni es la verdad de un momento histórico, sino donde el ser cultural produce verdades históricamente condicionadas y válidas.
\end{abstract}

Enrique Leff Zimermann (Leff, 2006:137)

Chaque époque apporte sa loupe ou son filtre; le temps masque ou déforme; le temps aussi redéfinit et redécouvre. Au début du siècle dernier la rhétorique de la modernité paraissait avoir consolidé, de part et d'autre de l'Atlantique, le triomphe de Caliban, personnage mythique et contradictoire de La tempête de Shakespeare, interprété tantôt comme le symbole du matérialisme anglo-saxon et de l'homogénéisation culturelle, tantôt comme le porte-parole des peuples colonisés et périphériques. Il incarnait ainsi les deux faces d'une même pièce: d'un côté l'implacable pouvoir de la raison technique et marchande, de l'autre, la force irrésistible de l'utopie (Darío 1950, Rodó 1991, Vior 2000). Dans notre monde d'aujourd'hui, accéléré et fluctuant, l'incertitude et la complexité semblent avoir assombri l'horizon du progrès de telle sorte qu'il n'existe plus de rationalités indiscutables et transmissibles, capables de tout embrasser, de restreindre la diversité naturelle et sociale dans l'enceinte sûre et intelligible des concepts ou des laboratoires et de prévoir, même à moyen terme, l'évolution des phénomènes qui nous entourent (Valcárcel 2002, Innerarity 2011). Les modèles théoriques

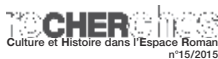


sont remis en cause et les pouvoirs, même démocratiques, perdent leur légitimité (Rosanvallon 2008), tandis que des pressions insoutenables s'exercent sur les populations et leurs environnements, soumis à la dictature du temps et des marchés, aux inégalités culturelles et sociales, aux politiques de surveillance et d'austérité (Cordera et al. 2008, Graham 2012, Rosa 2010).

L'histoire des peuples ibériques et latino-américains, dans leur rapport au monde occidental, a souvent été gouvernée par les contradictions et les équivoques, les emprunts et les rejets successifs. Les changements planétaires survenus ces dernières années semblent confirmer ces tendances, tout en renversant aussi les perspectives. Tandis que la mondialisation bouleverse l'ordre centrifuge et vertical qui garantissait depuis des siècles la pérennité des transferts et le maintien des dépendances, on observe aujourd'hui des manifestations récurrentes de réappropriation (sémantique, épistémique, politique, économique, sociale et culturelle) des espaces collectifs réels et symboliques. C'est ainsi que les crises, tout en suscitant le repli, l'indignation et la résistance, semblent resignifier aussi les perspectives de lêtre, du vivre ensemble et du possible, à travers des processus intellectuels de reconstruction autonome et de nouvelles formes d'agir sur le monde.

Explorer l'espace ibérique et latino-américain à partir de la grille de lecture qu'offre le concept de crise, c'est donc reprendre pour la mille et unième fois ce voyage interminable des identités qui se cherchent à travers la Méditerranée et l'Atlantique. C'est aussi mordre la pomme de l'Occident incontournable qui nous renvoie à nos pêchés originels. C’est dérouler le pli infini de la césure qui scinde le vers en deux hémistiches. C'est accepter de s'engager finalement sur un chemin sinueux et incertain dont nous ne pourrons parcourir ici qu'une brève étape, à la fois réelle et imaginaire, comme lors de ces promenades au cours desquelles se mêlent images, observations et rencontres fortuites à nos rêveries solitaires. Si la métaphore shakespearienne de La tempête peut encore rendre compte aujourd'hui des turbulences de notre époque et du caractère dynamique et symbolique de la relation paradoxale et signifiante de lêtre hispanique avec l'altérité, le texte de l'économiste et environnementaliste mexicain Enrique Leff Zimmermann, cité en exergue de cet article, nous servira de fil conducteur pour essayer d'analyser comment les obstacles et les crises induisent des changements paradigmatiques et produisent des re-significations qui illustrent les dérives et révèlent les contingences de toutes les vérités humaines.

\section{L'être en mouvement}

Ce qui caractérise le passage du temps, d'ailleurs, n'est-ce pas avant tout le changement? La continuité historique est toujours une construction culturelle qui relève bien plus d'une fiction, entretenue par des intérêts politiques, que d'un ensemble de données objectives scientifiquement vérifiables. Les événements, au contraire, se produisent dans le monde réel sous l'impulsion des mouvements pluriels et incessants des êtres et les transformations des contextes (Álvarez Junco 
2001). Par conséquent il convient de se demander si les crises ne seraient pas elles aussi d'abord, tout simplement, des constantes de l'être en mouvement.

En les considérant comme des phénomènes accidentels plutôt que comme des invariants de la condition humaine, ne sommes-nous pas en train de conforter, en outre, la nouvelle fable transhumaniste vers laquelle tout nous entraîne aujourd'hui: la mécanisation des savoirs (Zubiri 1963, Cerezo Galán 1985), l'insoutenable accélération technique (Rosa 2010), la liquéfaction des relations humaines (Bauman 2002) et l'imparable croissance économique (Cohen 2012). Tandis que les logiques anthropophages de la modernité occidentale s'intensifient à l’ère globale (Bauman 2007) l'humanité semble ellemême pétrifiée par l'immobilisme que lui confère la peur de sa propre vitesse. Cette double tension consistant à appuyer sur le frein et l'accélérateur en même temps, n'est-elle pas aussi, finalement, une métaphore de l'existence humaine? ¿Ser es estar en perpetua crisis?

Dans le cadre mondialisé, ubiquiste et réticulaire de ce début de $\mathrm{xxI}^{\mathrm{e}}$ siècle, que devient lêtre hispanique dans sa quête d'intimité collective (politique, sociale, culturelle)? Est-il en mesure aujourd'hui de développer les outils lui permettant de surmonter ses propres obstacles circonstanciels et générationnels et d'accéder un jour, non pas à une nouvelle et hypothétique utopie, mais à une resignification valable et acceptable de son être en devenir et en dialogue permanent avec l'altérité? Cette question nous invite à adopter d'emblée vis-à-vis du temps, des individus et de l'espace, une perspective oblique, plurielle et non linéaire qui nous aide aussi à envisager, non pas les identités et leurs problématiques souvent figées et insolubles, mais les interfaces et les relations qui les transforment, les dynamisent et les régénèrent. Il s'agit donc d'aborder, plutôt que les entités elles-mêmes, les fragments divers qui les constituent, plutôt que les êtres en euxmêmes, ce qu'il y a entre eux, ces dimensions gestuelles, affectives, linguistiques, difficilement saisissables mais autour desquelles se génèrent pourtant les langages et les récits existentiels.

L'histoire qui nous occupe ici est riche de ses mouvements divers et multidirectionnels, de la circulation des êtres, des savoirs et des imagi-naires: découvertes, inventions, conquêtes, colonisations, migrations, exils, transferts, représentations, impositions, assimilations, chocs, ruptures, émancipations, etc. Elle se renouvelle ainsi, en permanence, de l'intérieur et depuis l'extérieur, comme dans le fonctionnement autopoiétique des êtres vivants. Elle se compose, se décompose et se recompose alternativement aussi, depuis les perspectives de la distance géographique, culturelle ou historique. Il s'agit d'une distance tantôt réelle ou fictionnelle comme dans le Journal de bord de Christophe Colomb ou dans les Lettres Marocaines de José Cadalso, et qui répond à un jeu de miroirs et de mémoires tantôt concaves ou convexes comme dans les Lumières de Bohème de Valle-Inclán. Léloignement souvent rapproche, la proximité parfois déforme. Alejo Carpentier décrivait ainsi sa prise de conscience des réalités latino-américaines depuis son exil parisien: «Empecé a verlo mejor, ciertas cosas cuya existencia no advertía, por tenerlas demasiado cerca, empezaron a 
cobrar enormes relieves para mí cuando pude contemplarlas a distancia, con la suficiente perspectiva » (Palma 1991 : 20). C'est à Londres que Juan Pablo Viscardo écrivit en 1792 sa célèbre Lettre aux Espagnols Américains (Viscardo 1977) et à New-York que José Martí rédigea un siècle plus tard son essai Nuestra América (Martí 2005). C’est depuis leur exil américain que Claudio Sánchez Albornoz, María Zambrano, León Felipe ou Luis Buñuel (parmi tant d’autres) entreprirent de décrypter les ambigüités de lâme espagnole. La distance nous permet de mieux distinguer cette partie de nous-mêmes que nous narrivons jamais à saisir justement parce quelle avance en même temps que nous et qu'il nous manque toujours le recul nécessaire pour l'appréhender. Les crises douloureuses et souvent répétitives auxquelles ont été confrontés les peuples ibériques et latinoaméricains ne viendraient-elles pas justement de cette difficulté à prendre du recul pour saisir la complexité des mouvements d'ensemble? C'est après coup, seulement, que le voile se lève sur une réalité a priori imprévisible et nébuleuse et que se matérialise l'enchaînement des causes et des conséquences. C’est le mouvement qui suscite la crise mais la sortie de crise suppose donc aussi, conformément à cette dynamique, un déplacement. C'est ce qui fait dire à Ignacio Escolar, à propos de la situation critique de l'Espagne depuis 2008, que sortir du tunnel ce n'est jamais revenir en arrière mais arriver dans un autre lieu:

La crisis económica es como una sequía que ha dejado el embalse sin agua hasta dejar visible el fondo del pantano: la basura que la lluvia de los buenos años ocultó. Ha derivado en una crisis institucional que corroe las bases del contrato social. Algún día se saldrá del túnel, pero esta metáfora se suele interpretar mal. Muchos creen que volveremos a la luz por el mismo sitio por el que entramos, que será un regreso a la España de 2007. Pero del túnel siempre se sale a otro lugar. (Escolar 2012: 14).

\section{Le sentiment de soi, le miroir de l'autre}

Comment atteindre la lumière au bout du couloir obscur? Se trouve-t-elle à l'intérieur ou bien à l'extérieur? Faudra-t-il partir ou renoncer à être soimême pour continuer d'exister? La célèbre déclaration de 1910 de José Ortega y Gasset, «España era el problema y Europa la solución» (Ortega y Gasset 1990: 62), a suscité récemment de nombreuses discussions dans la presse espagnole et sur Internet ${ }^{1}$. Lenthousiasme europhile des premières générations du postfranquisme a cédé la place dans ce pays à un eurosceptisme désabusé, échaudé par les dérégulations sociales et les coupes budgétaires drastiques imposées par Bruxelles au gouvernement de Madrid. Tandis que le mouvement des Indignés continuait de répéter au printemps 2012 que le problème ne venait pas des populations victimes de la récession mais du système de fonctionnement luimême de l'économie et des institutions, le président du gouvernement, Mariano Rajoy, laissa échapper dans les médias le message «España no es Uganda» qui

1 Carlos Elordi, Europa ya no es la solución, sino otro problema, eldiario.es, 30/10/2012 
suscita alors de vives réactions de protestation nationales et internationales ${ }^{2}$. Ces deux postures, la première cherchant à s'attaquer aux origines politiques et intellectuelles de la crise, la seconde, très maladroitement, aux représentations culturelles, nous renvoient aux débats historiques sur les rapports conflictuels du monde hispanique à ses altérités de voisinage ou d'origine ou sur l'impossibilité d’adapter ce que José Luis Abellán appelait «los mitos fundantes de sentido y cultura» (Abellán, 1979: 23) aux logiques de la raison occidentale.

D’après Américo Castro la spécificité de la culture espagnole, par rapport aux autres cultures européennes qui ont privilégié l'idée de progrès, les valeurs matérielles (prospérité, production, richesse) et le développement de la raison pure (formalisme logique, sciences et technologies), tient à ce quelle a toujours considéré d'abord l'être humain comme une réalité nue et absolue et, plus accessoirement ensuite, les produits à travers lesquels celui-ci tente de substituer la conscience de son existence. En d'autres termes, pour les Espagnols, les individus seraient plus intéressants par ce qu'ils sont réellement que par leurs productions ou la fonction sociale qu'ils représentent (Castro 1977, Abellán 1989: 22). C'est ce qui pourrait expliquer la résistance des intellectuels ibériques aux paradigmes de la raison instrumentale et la recherche de voies alternatives telles que la raison vitale d'Ortega y Gasset (Ortega y Gasset 1987), la raison poétique de María Zambrano (Zambrano 2001) ou la «inteligencia sentiente» de Xavier Zubiri (Zubiri 1980).

Plus largement, le monde hispanique (dans son acception intercontinentale et interculturelle) s'est régulièrement trouvé relégué, au cours de l'histoire, dans les marges du monde occidental dont les frontières ont été d’abord pyrénéennes avant dêtre océaniques. Antonio Machado, en pleine guerre civile, exprimait ainsi, dans les pages du journal La Vanguardia, son amertume vis-à-vis d'une Europe ingrate et amnésique envers la nation méditerranéenne qui avait contribué pourtant, des siècles durant, à la sauvegarde des intérêts continentaux:

España ha sido víctima de las mayores calumnias; porque hasta el título de europea se le ha negado. Quienes, con total desconocimiento de la historia y de la geografía, sostienen que el África empieza en los Pirineos, olvidan que en los Pirineos no empieza sino que en ella acaba el gran baluarte de la Europa occidental, erizado de sierras y poblado de pechos indomables, merced a los cuales Europa es Europa. (Machado 1983: 290)

De l'autre côté de l'Atlantique, les auteurs latino-américains n'ont pas manqué de relever, non plus, l'indifférence notoire de l'Europe à l'égard des productions intellectuelles d'un sous-continent qui avait été condamné par Hegel à une sorte de non-existence civilisationnelle. L’Amérique Latine, d’après le philosophe allemand, nétait pas un être, elle n'avait ni originalité ni sens historique et se trouvait encore dans la préhistoire (Hegel 1972, Dussel 1973, Soliz Rada 2008). Les Espagnols, eux-mêmes, tout en célébrant, comme José Alcalá

2 Pablo Pardo, Bronca mundial en Internet por el "Aguanta, España no es Uganda", elmundo.es, 13/06/2012. 
Galiano, l'intimité philologique, intellectuelle et littéraire qui unissait les peuples hispaniques, "par des affinités et des attirances supérieures à celles qui régissent la fausse mécanique de la politique internationale» (Alcalá Galiano 1892: 546), continuèrent de montrer, après les indépendances, une sorte de condescendance postcoloniale à l'égard des jeunes républiques d'outre-Atlantique. Ils évaluèrent leurs lointains cousins d’Amérique à travers le prisme déformant de leurs rancœurs, comme «des êtres ignorants, voire infantiles, dotés des plus grands vices et défauts et composant des sociétés chaotiques, très inférieures à celles de l'époque coloniale» (Rama 1982: 90-91). Cette attitude ne fut pas exempte de préjugés raciaux que les élites créoles répercutèrent à leur tour sur leurs populations d'origine indienne et africaine (Martí 2005, González Prada, 1904). C'est pourquoi Sarmiento réclamait pour l'Argentine, en 1884, une émigration destinée à "corriger le sang indigène par du sang européen" (Sarmiento 1997: 192) et presque un siècle plus tard encore, son compatriote, le philosophe Alberto Caturelli affirmait encore que si l'Europe était le continent de l'être ouvert et total fécondé par l'esprit, l'Amérique au contraire était celui de lêtre brut, encore non fécondé par l’esprit (Caturelli 1956-1957, Rolland 1998: 405).

Héritiers d'une identité espagnole déjà diverse et morcelée, issue de la fermentation des langues et cultures européennes et méditerranéennes, les Latino-américains se sont trouvés confrontés à des problématiques identitaires encore plus complexes, que Leopoldo Zea exprimait en ces termes au milieu du siècle dernier:

Lo nuestro, lo propiamente americano, no está en la cultura precolombina. ¿Estará en lo europeo? Ahora bien, frente a la Cultura Europea nos sucede algo raro, nos servimos de ella pero no la consideramos nuestra, nos sentimos imitadores de ella. Nuestro modo de pensar, nuestra concepción del mundo, son semejantes a los del europeo. La Cultura Europea tiene para nosotros el sentido de que carece la cultura precolombina. Y sin embargo, no la sentimos nuestra. (Zea 1942: 66)

Aujourd'hui, alors que la mondialisation (discontinue, latérale et réticulaire) semble déliter les représentations mentales et les structures qui assuraient autrefois la cohésion des ensembles nationaux et identitaires (Bauman 2002), l'affirmation d'une intimité collective du monde hispanique est-elle toujours de mise? De plus en plus de personnes pensent que la quête de soi n'est pas tant une recherche de ce que l'on est qu'une quête de ce que l'on désire. C'est ce qui fait dire au philosophe Miguel Rojas Mix que les racines de l'identité se trouvent dans le futur. Il y a deux manières d'appréhender la question de l'identité, selon lui: une modalité prospective, qui l'envisage à partir d'une image sociale en devenir; ou bien une démarche archéologique fondée sur une vision immanentiste. Dans le premier cas il s'agirait de construire l'identité, dans le second cas de la découvrir, comme s'il était question d'un être préexistant, d'une vérité en soi. L'une (utopique) et l'autre (uchronique) de ces approches, d'après l'auteur, sont insuffisantes et pernicieuses (Rojas Mix 1991: 383-400). On retrouve cette même dualité janusienne, lorsqu’on passe de l'identité à la nationalité. Grínor Rojo estime pour sa part que lémergence du concept moderne de nation s'est 
opérée dans le cadre d'une superposition ou coexistence de deux sortes de cultures, celle de la nation de "provenance et/ou de destin» et celle de la nation des «citoyens» (les natio et civitas de l'empire romain). L'une appréhende la nationalité comme l'expression de l'appartenance à des communautés d'origine, intégrées géographiquement par la proximité et la colonisation et culturellement par la langue, les coutumes et les traditions collectives mais pas nécessairement structurées autour d'une organisation étatique. L'autre conçoit la nationalité comme un modèle d'intégration citoyenne qui doit s'appuyer sur la pratique d'une souveraineté populaire intersubjective, tolérante envers les différences et «auto-législative» (Rojo 2006: 32-34).

Quoi qu'il en soit, les notions d'identité et de nationalité ne coulent jamais de source. Ce sont des représentations ou des constructions de la réalité, des fictions destinées à créer des liens entre les individus, à les protéger d'eux-mêmes et d'éventuels dangers extérieurs, à intégrer mais aussi à exclure, à échanger mais aussi à renfermer, à contenir. Dans nos sociétés occidentalisées, quels rôles jouent ou peuvent jouer encore les identités issues des nations ibériques et américaines pré-modernes, les États-nations qui se sont forgés de part et d’autre de l'Atlantique depuis le $\mathrm{XIX}^{\mathrm{e}}$ siècle et les supranationalités héritées de l'histoire coloniale ou remodelées par les échanges internationaux et les processus d'intégration régionaux de lère globale? Que veut dire être latino-américain ou basque lorsqu'on vit en Espagne en ce début de $\mathrm{xxI}^{\mathrm{e}}$ siècle? Quelle place devrait-on accorder aux nations quechua ou guarani dans l'Unasur? Dans quelles mesures la langue espagnole, relativement homogène bien que planétaire, peutelle constituer, de nos jours, un facteur de cohésion capable d'interconnecter des peuples, des sociétés, des cultures et des économies, de favoriser leur épanouissement tout en limitant leur nuisance ou leur vulnérabilité?

\section{«Del desencanto al desenmascaramiento»}

Nuisance et vulnérabilité: ces deux termes apparaissent au sortir du tunnel de la modernité. Toute formulation politique, économique ou philosophique qui ne prenne pas en compte ces deux paramètres semble condamnée à l'échec (Innerarity 2011 : 135, Leff 2007: IX-XXI). D’après le philosophe Pedro Cerezo Galán, l'époque actuelle ressemble en bien des points à la période charnière de la prémodernité, à cheval entre le Moyen-Âge et la Renaissance, celle de Montaigne qui dépeignait dans ses Essais un monde d'immédiateté peuplé d'objets troubles et chancelants (Montaigne 1965). Nous vivons, selon Cerezo Galán, une période de mal-être et de faillite des institutions qui découle de la crise de la raison occidentale, laquelle dérive à son tour d'une mathématisation excessive de la pensée qui s'est émancipée de la vie naturelle et sociale. La perte de contact avec le sol nourricier a provoqué l'indépendance de la raison mais aussi et surtout son déracinement par rapport au contexte vital et social dans lequel elle a été produite (Cerezo Galán 1985, Leff 2007). Pour Xavier Zubiri, c'est le développement gigantesque de la technique qui a changé profondément 
la manière dont vivent les êtres humains. Le passage de l'homo sapiens à l'homo faber, déterminé par la science moderne, a suscité une crise qui affecte l'idée même de monde et la maitrise qu’ont les individus de leur propre existence. (Zubiri 1963: 28-29, Fornet-Betancourt 2009: 14). Amelia Valcárcel, reprend à son compte la formulation wébérienne de «désenchantement» pour expliquer l'émergence au $\mathrm{XVII}^{\mathrm{e}}$ siècle, après les traités de Westphalie, de la modernité occidentale dont les deux caractéristiques fondamentales ont été, selon elle, la laïcisation et la sécularisation, c'est-à-dire la substitution des textes sacrés par la science pour l'explication du monde (sa composition, sa structure, son origine) et le remplacement de l'idée religieuse du destin céleste par le concept du progrès linéaire (Valcárcel 2002: 104-105). C’est cette même évolution qu’Ortega y Gasset avait décrite de son côté comme la transition de la cosmologie vers la cosmométrie (Fornet-Betancourt 2009: 11).

Quels que soient les termes utilisés, il semble bien, toutefois, que l’entrée du monde hispanique et son maintien dans la modernité occidentale ne se soient pas faits sans ambigüités ni sans heurts. D’autres formes de pouvoir et de hiérarchies, politiques, religieuses, sociales, raciales ont toujours altéré ou entravé, depuis le début, les logiques de la rationalité mathématique ou de l'utilitarisme marchand (Grosfoguel 2012). De même que les crises peuvent être considérées comme des constantes de lêtre en mouvement, la double-vérité semble avoir été une constante de l'histoire de la modernité. Sans elle, comment concilier en effet les exigences de la foi et celles de la raison, les entreprises de découverte et les mécanismes d'invention et de dissimulation bien connus des historiens, les motivations de l'évangélisation des Indiens et celles de l'esclavage?

Si le désenchantement du monde à la Renaissance, c'est-à-dire la relégation du magique et du surnaturel par la raison, a fait éclore la modernité, c'est le desengaño, d’après Valcárcel, qui ouvre le cycle de la postmodernité (Valcárcel 2013). En proclamant la mort des grands récits et la fin de l'histoire, Lyotard (1979) et Fukuyama (1993) ont-ils ouvert une nouvelle boîte de Pandore? Peut-on vraiment échafauder une nouvelle modernité post-occidentale, voire post-rationnelle, capable de surmonter les crises du présent sans hypothéquer l'avenir? De nombreux intellectuels coïncident désormais sur l'idée que le temps a supplanté l'espace (Rosa 2010). Pour Daniel Innerarity, toute théorie de la société devrait être aujourd'hui « une théorie du temps et plus particulièrement de l'usage que nous faisons du futur». Soumis à la tyrannie de l'instant, de l'immédiateté, de l'urgence, nous avons fini par considérer l'avenir comme la poubelle dans laquelle nous rejetons les problèmes non-résolus du présent (Innerarity 2008: 12, 38). Chaque époque recherche ses propres métaphores pour s'auto-définir. Deux concepts contradictoires, et dans une certaine mesure concurrents, connaissent aujourd'hui un franc succès: l'économie de la connaissance et la société du risque. Dans les deux cas il s'agit de formulations biaisées favorisant un point de vue finalisé sur le monde qui en restreint les potentialités (Citton 2010, Ballesteros Pérez 2013). Les enjeux collectifs peuvent-ils être sérieusement pris en compte sans passer par un dialogue intersubjectif permettant de confronter les diverses 
cultures d'interprétation? Pour l'environnementaliste mexicain Enrique Leff, il est plus important d'abord de redécouvrir les valeurs de médiation à travers une éthique de l'altérité plutôt que de définir une unité-valeur réductrice et stigmatisante (Leff 2007: XIX).

Mais toute construction exige souvent d'abord une déconstruction. Tandis que certains intellectuels explorent les soubassements de l'identité espagnole pour mieux la démystifier (Álvarez Junco 2001, Vernet 1999), d'autres penseurs hispaniques d'outre-Atlantique (Santiago Castro Gómez, Walter Mignolo, Aníbal Quijano, Alberto Moreiras, Ramón Grosfoguel, etc.) se sont donnés pour tâche, depuis des années, d'interroger dans leurs fondements les modèles de pensée occidentaux, parvenant à la conclusion que les rationalités qu'ils développent ne sont ni universelles ni irrévocables et qu'il est toujours possible, par conséquent, d'ouvrir la voie à d'autres possibles. C'est ainsi qu'ils ont forgé le concept de colonialité $d u$ savoir qui associe sur un même plan épistémologique l'histoire du colonialisme et celle de la modernité eurocentrique qui en a été le moteur et la justification. Ils ont cherché à montrer, par exemple, comment depuis le XVII ${ }^{\mathrm{e}}$ siècle (Descartes, Spinoza, Locke, Newton...) l'Occident a élaboré et formalisé un mode de production des connaissances qui répondait directement aux besoins cognitifs du capitalisme (la mesure, la quantification, l'objectivation du connaissable par rapport au connaisseur) pour le contrôle des relations entre la nature et les individus, dans la perspective de la propriété des ressources de production (Quijano 2000). Cela permet de déduire que léconomie de la connaissance ou le capitalisme cognitif ne sont pas des concepts nés de la dernière pluie. Au cours d'une conférence prononcée à Madrid en octobre 2012, le sociologue portoricain Ramón Grosfoguel rappelait que l'université occidentalisée (c’est-à-dire celle qui existe dans le monde entier aujourd'hui) privilégie depuis toujours une connaissance produite par des hommes (et non des femmes) de seulement six pays du monde, ce qui veut dire, en fin de compte, que l'expérience historicosociale de $6: \%$ de la population mondiale prétend rendre compte de l'être, du penser et de l'agir de l'humanité tout entière (Grosfoguel 2012). À cela s'ajoute aujourd'hui le sentiment d'une profonde crise de confiance épistémologique due au fait que les promesses qui ont légitimé le privilège de la connaissance scientifique occidentale (paix, rationalité, liberté, égalité, progrès, répartition des bénéfices de la croissance, etc.) ne se sont toujours pas réalisées. Outre les epistémicides qu'elle a provoqués de tout temps, dans la plupart des régions du globe, la rationalité occidentale n'a pas démontré, non plus, qu'elle était capable de contenir ses propres propensions eco-destructives et socio-destructives (Sousa Santos, 2005: 11-12). De fait, il semble de plus en plus évident aujourd'hui, pour les défenseurs de la diversité épistémologique du monde, qu'il existe une sorte de relation proportionnelle entre le progrès scientifique conduit par le monde occidental et la détérioration socioculturelle et environnementale. 


\section{Iberalternatives}

Les situations de crise ne sont peut-être pas les plus propices pour mettre à l'épreuve les concepts par lesquels nous essayons de les interpréter (Innerarity 2011: 135). Les véritables solutions sont souvent celles qu'on a tendance à esquiver parce qu'elles offrent plus de résistance (Ortega y Gasset 1999: 9). Des voix se lèvent, toutefois, de part et d'autre du monde hispanique pour proposer des changements paradigmatiques capables de surmonter ces obstacles. Au Mexique, Enrique Leff propose une nouvelle «rationalité environnementale» qui postule la nécessité d'instaurer des modes de production et des styles de vies en fonction des potentialités écologiques de chaque région, de la diversité ethnique et de la capacité de chaque population à autogérer ses ressources naturelles et ses processus productifs (Leff 2007). En Espagne, le mouvement des Indignés, réinvente de nouvelles formes d'expression politiques et gestuelles pour reconstruire le dialogue collectif. Dans nos sociétés violentes et désorientées, caractérisées par l'individualisme et l'exclusion, la rue redevient souvent un espace de médiation et de socialisation où se manifestent les peurs et les inquiétudes, mais aussi le talent, la créativité et ou l'expression fait littéralement exister la signification (Sánchez Albarracín 2012). Dans les pays Andins, le concept traditionnel du «Buen Vivir» permet de redonner du sens à des projets collectifs intégrateurs qui renoncent à une problématisation anthropocentrique des enjeux de convivialité (Luna Rangel 2010). En marge des sommets ibéro-américains où se développent des stratégies politiques et marchandes orchestrées par les États, des mouvements culturels et sociaux altermondialistes promeuvent des expériences participatives transcontinentales. Dans les universités européennes et latino-américaines des centaines de chercheurs se sont lancés récemment dans un vaste projet d'histoire conceptuelle, "Iberconceptos», dont le but est "d'étudier, de manière systématique, sur le long terme et dans une perspective comparée, les concepts, langages, vocabulaires, discours et métaphores politiques les plus importants, en circulation dans le monde ibéro-américain au cours de ces derniers siècles». Il s'agit de fonder « une histoire politico-intellectuelle euroaméricaine, qui, dans l'avenir, avec d'autres histoires régionales correspondant à d'autres grandes aires géoculturelles en contact, pourrait trouver sa place dans une histoire globale, ou, si l'on veut, véritablement universelle» ${ }^{3}$.

Lêtre humain, dès l'origine de son espèce, nous dit le philosophe Enrique Dussel, n'existe pas tout seul. Il se trouve toujours plongé dans une intersubjectivité de fait. Sa pensée est dialogique, elle a besoin d'un je et d'un $t u$, et ne peut sétablir que dans l'horizon d'un nous. La langue, les gestes, les institutions sont les exemples les plus significatifs de ces interfaces qui relient, modèlent et transforment les individus et leurs contextes au gré du temps (Dussel 1973: 68). L'une des conditions fondamentale du langage n'est-elle pas précisément le consensus? (Echeverría 2011: 50) Je dialogue, donc je suis. Ma raison devrait être

3 Présentation du projet disponible sur le site Internet: http://www.iberconceptos.net 
aussi la raison de l'autre, mise en partage. Je suis d'ailleurs moi-même le résultat d'une percolation d'altérités.

Les risques et les incertitudes qui nous désorientent aujourd'hui sont peutêtre les lambeaux que nous a laissés la tempête de la modernité occidentale après son passage dévastateur sur le monde. Il est hasardeux toutefois de penser que les recettes d'autrefois, même désenchantées, pourraient nous guider vers la sortie du tunnel. Léchec de Caliban, ce n'est ni l'échec du matérialisme ni celui de l'idéalisme, mais celui de notre acharnement à refuser la dérive du temps et la pluralité des êtres.

\section{Bibliographie}

Abellán, J. L., 1979, Historia crítica del pensamiento español, tomo 1, Madrid, Espasa-Calpe.

Abellán, J. L., 1989, El Pensamiento español contemporáneo y la idea de América, Madrid, Anthropos.

Alcalá Galiano, J., 1892, Acerca de los servicios que, en el desempeño de su cargo, pueden prestar los cónsules para mayor seguridad del comercio de libros y obras artísticas, y planteamiento del giro consular entre los estados hispanoamericanos y España, Congreso Literario Hispano-Americano, Madrid, Edition fac-similé, Madrid, Instituto Cervantes, 1992, p. 545-556.

Álvarez Junco, J., 2001, Mater Dolorosa, La idea de España en el siglo XIX, Madrid, Taurus, 2001.

Ballesteros Pérez, C., 2013, La Sociedad del Riesgo como Profecía Autocuplida in: Arroyo Pichardo G. (coord.), Lo Local y lo Global en las Relaciones Internacionales, México, UNAM, p. 31-43.

Bauman, Z., 2002, Modernidad Liquida, México, FCE.

Bauman, Z., 2007, Le présent liquide: peurs sociales et obsession sécuritaire, Paris, Seuil.

Castro, A., 1977, An Idea of History, Selected essays of Américo Castro, Columbus, Ohiao State U.P.

Caturelli, A., 1956-1957, América Bifronte, Buenos Aires, Ed. Troquel SA.

Cerezo Galán, P., 1985, La crisis de la razón occidental, Conférence, Madrid, 28/02/1985, Fundación Juan March, disponible sur http://www.march.es.

Citton, Y., 2010, L'avenir des Humanités, Economie de la connaissance ou cultures de l'interprétation, Paris, La Découverte.

Citton, Y., 2012, Renverser l'insoutenable, Paris, Seuil.

Cohen, D., 2012, Homo Economicus: Prophète égaré des temps nouveaux, Paris, Albin Michel.

Cordera, R., Ramírez Kuri, P., Ziccardi, A., 2008, Pobreza, desigualdad y exclusión en la ciudad del siglo XXI, México, Siglo XXI. 
Darío, R., 1950, El Triunfo de Calibán, El Tiempo, Buenos Aires, 20/05/1898 / El Cojo Ilustrado, Caracas, 1/10/1898, in: Obras Completas, tomo IV, Madrid, Afrodisio Aguado, p. 569-576.

Dussel, E., 1973, América Latina: dependencia y liberación, Buenos Aires, Fernando García Cambeiro.

Echeverria, R., 2011, Ontología del lenguaje, Buenos Aires, Granica.

Fornet-Betancourt, R., 2009, «Filosofía y ciencia en el mundo hispánico: una meditación intercultural de la ciencia», Logos: Revista de Filosofía, vol. 37, $\mathrm{n}^{\circ} 111$, p. 41-56.

Fukuyama, F., 1993, La Fin de l'Histoire et le dernier homme, Paris, Flammarion.

González Prada, M., 1904, Nuestros Indios, in: Cuadernos de cultura Latinoamericana, 29, México, Unam, 1978.

Graham, S., 2012, Villes sous contrôle, Paris, La Découverte.

Grosfoguel, R., 2012, Crisis financiera, colonialidad del poder y la Europa del Sur, 19 octubre de 2012, UAM, Madrid.

Hegel, G. W.F., 1972, Vorlesungen über die Philosophie der Geschichte, in: Werke in zwanzig Bänden. Frankfurt am Main: Suhrkamp Verlag, vol. 12.

Innerarity, D., 2008, Le futur et ses ennemis - De la confiscation de l'avenir à l'espérance politique, Paris, Climats.

Innerarity, D., 2011, La democracia del conocimiento, Barcelone, Paidós.

Leff Zimermann, E., 2006, Aventuras de la epistemología ambiental, México, Siglo XXI.

Leff Zimermann, E., 2007, Racionalidad ambiental: la reapropiación social de la naturaleza, México, Siglo XXI.

Luna Rangel, M., 2010, El Origen del concepto del Buen Vivir o Vivir Bien, Aporrea, 1/10 2010.

Lyotard, J.-F., 1979, La Condition postmoderne: rapport sur le savoir, Paris, Éditions de Minuit.

Machado, A., 1983, La Guerra. Escritos: 1936-39, édition, introduction et notes par Julio Rodríguez Puértolas y Gerardo Pérez Herrero, Madrid, Emiliano Escolar Editor.

Martí, J., 1891, Nuestra América, Caracas, Fundación Biblioteca Ayacucho, 2005.

Montaigne, M. (de), 1965, Les Essais, livre III (extraits), chap. 2 «du repentir», Larousse.

Ortega y Gasset, J., 1987, El tema de nuestro tiempo, Madrid, Espasa Calpe, «Austral».

Ortega y Gasset, J., 1990, La pedagogía social como programa político, Discursos políticos, Alianza Editorial, Madrid.

Ortega y Gasset, J., 1999, «Prólogo» a la cuarta edición, España Invertebrada, Universidad de Extremadura, Servicio de Publicaciones.

Palma, M., 1991, El Mito de París, Entrevistas con Escritores Latinoamericanos en París, Indigo \& Côté-femmes éditions. 
Quijano, A., 2000, Colonialidad del poder y clasificación social, Journal of worldsystems research, Summer / Fall.

Rama, C., 1982, Historia de las relaciones culturales entre España y América latina. Siglo XIX, México-Madrid, Fondo de Cultura Económica.

Rodó, J. E., 1991, Ariel, Madrid, Espasa Calpe, «Austral».

Rojas Mix, M., 1991, Los cien nombres de América: eso que descubrió Colón, Barcelone, Editorial Lumen.

Rojo, G., 2006, Globalización e identidades nacionales y postnacionales... ¿de qué estamos hablando?, Santiago de Chile, LOM Ediciones, «Escafandra».

Rolland, D., 1992, «Les perceptions de la France en Amérique Latine: structures et évolution, 1918-1945», Mélanges de la Casa de Velázquez, vol. 28, p. 161-189.

Rosa, H., 2010, Accélération, Une critique sociale du temps, Paris, La Découverte.

Rosanvallon, P., 2008, La légitimité démocratique, Éditions du Seuil.

Sánchez Albarracín, E., 2012, «La rhétorique de l'illégitimité de la représentation politique de l'Argentine vers l'Espagne», in: Fourtané M., Guiraud M. (éd.), Emprunts et transferts culturels: du monde luso-hispanophone vers l'Europe, PUN, Presses universitaires de Lorraine, p. 337-358.

Sarmiento, D.F., 1997, Conflicto y armonía de las Razas en América, Segunda Parte póstuma, 1888, in: Botana N. R., Gallo E., De la República posible a la República verdadera (1880-1910), Biblioteca del Pensamiento Argentino, III, Buenos Aires, Espasa Calpe Argentina, p. 187-195.

Soliz Rada, A., 2008, Hegel, Marx y América Latina, 19-10-2008, disponible sur http://www.aporrea.org

Sousa Santos B. de, 2005, Introducción General, in: Sousa Santos B. de, García Villegas M., et al., Emancipación Social y violencia en Colombia, Bogotá, Ed. Norma, p. 11-29.

Valcárcel, A., 2002, Ética para un mundo global, Una apuesta por el humanismo frente al fanatismo, Madrid, Temas de Hoy.

Valcárcel, A., 2013, Cómo vivir en un mundo global y desengañado, Conférence, Madrid, 19/03/2013, Fundación Juan March, http://www.march.es

Vernet, J., 1999, Lo que Europa debe al Islam de España, Barcelona, El Acantilado. Vior, E. J., 2000, «Visiones de Calibán, visiones de América», CUYO, Anuario de Filosofía Argentina y Americana, no 17, p. 89-103.

Viscardo, J.P., 1977, Carta a los españoles americanos (1792), in: Pensamiento político de la emancipación (1790-1825), vol.1, Fundación Biblioteca Ayacucho, p. 51-58.

Zambrano, M., 2001, Claves de la razón poética, Madrid, Trotta, Estructuras y Procesos, Filosofía.

Zea, L., 1942, «En torno a una filosofía americana», Cuadernos Americanos, México, 3, mayo-junio 1942, p. 63-78.

Zubiri, X., 1963, Nuestra Situación Intelectual, in: Naturaleza, Historia, Dios, Madrid. http://www.zubiri.org/works/spanishworks/nhd/nuestrasituacion.htm 
Zubiri, X., 1980, La intelección humana: Inteligencia sentiente, Madrid, Alianza. 